\title{
Esophageal Cancer: Current Options for Therapeutic Management
}

\author{
Li Sun Hongwei Zhang Kaichun Wu \\ Xijing Hospital of Digestive Diseases, Xijing Hospital, Fourth Military Medical University, \\ Xi'an, China
}

\section{Key Words}

Esophageal cancer · Therapeutic management · Surgery · Radiotherapy · Chemotherapy

\begin{abstract}
Background: Esophageal cancer (EC) is the eighth most common cancer worldwide. A worldwide-established consensus on therapeutic pathways for EC is still missing. Debate exists on whether neoadjuvant and adjuvant treatment regimens improve the prognosis and which surgical approach reaches objective benefits.

Summary: This article discusses the appropriate option of the current different curative treatments in patients with EC, including surgical treatment and adjuvant therapy.

Key Message: To maximize survival and quality of life and also decrease postoperative complications, the present recommended therapeutic management of EC should be individualized multidisciplinary team approaches according to patients' staging and physiologic reserve.

Practical Implications: The aim of this article is to provide a decision support and also a discussion based on clinical therapeutic strategy in order to characterize the beneficial approach which reaches an optimal balance between radical resection, postoperative outcome and long-term survival of EC.

(C) 2014 S. Karger AG, Basel
\end{abstract}

\section{Introduction}

Esophageal cancer (EC), including squamous cell carcinoma (SCC) and adenocarcinoma (AC), is the eighth most common cancer worldwide. It affects more than 450,000 people worldwide and the incidence is increasing rapidly [1]. It is the sixth leading cause of cancer- 
related mortality because of the aggressive nature and poor survival rate [2, 3]. According to data from surveillance, epidemiology and end results throughout the last 30 years, 5-year survival rates for EC have improved poorly from 5\% between 1975 and 1977 to 19\% between 2001 and 2008 [4]. The treatment of EC has been debated controversially over the past decades. Current clinical therapeutic management for EC is individualized according to the patient's disease staging, histology and physiologic reserve. The outcome of patients might be influenced by different approaches. A worldwide-established consensus on therapeutic pathways is still missing. Debate exists on whether neoadjuvant and adjuvant treatment regimens improve the prognosis and which surgical approach reaches objective benefits [5, 6].

This article discusses the appropriate option of the current different curative treatment in patients with EC. The aim of this article is to provide a decision support and also a discussion based on clinical therapeutic strategy in order to characterize the beneficial approach which reaches an optimal balance between radical resection, postoperative outcome and long-term survival.

\section{Endoscopic Treatment}

Early esophageal cancer (EEC) involving the epithelium or the lamina propria is a candidate for endoscopic therapies including endoscopic mucosal resection and endoscopic submucosal dissection, because nominal lymph node metastasis has been reported in EEC confined to these layers [7]. Staging (TNM) must be confirmed before endoscopic therapy is started. Depth of invasion and other tumor characteristics, such as length, differentiation and angiolymphatic invasion, should be assessed in addition to nodal (N) and metastatic (M) status [1]. For EEC invading the muscularis mucosa, the lymph node metastasis rate is reported as 9\%, and for cancer with minute submucosal invasion the rate increases to $19 \%$ [8]. Therefore, for patients unwilling to undergo esophagectomy or for patients with severe comorbidity, endoscopic treatment may be a first choice in muscularis mucosa or slightly submucosal invasive cancer [9].

Ell et al. [10] used endoscopic mucosal resection and photodynamic therapy to treat 100 highly selected patients who had T1 intramucosal cancer and reported an estimated 3-year survival of $98 \%$. These results are encouraging, but the resection margins were positive in around one third of patients, and recurrent or metachronous lesions were detected in $11 \%$ of patients during a median follow-up of 33 months. In a study of resection of T1 tumors, multifocal neoplasia, angiolymphatic invasion or nodal metastases were frequently noted irrespective of tumor depth, which led the authors to conclude that endoscopic therapies should be reserved for high-risk patients [11]. EEC spreading to more than three quarters of circumference of the esophagus are not absolutely indicated even if the invasion depth is limited to the epithelium or lamina propria [7]. Although such lesions can be removed endoscopically, there are concerns of complications including postprocedural stricture [12-14]. Further work is required to define the role of endoscopic therapies with curative intent for EC.

\section{Surgical Treatment}

\section{Resection}

Surgical resection has been the gold standard for localized EC for decades. However, due to a poor 5-year survival rate, which is approximately $36 \%$ in patients undergoing esophagectomy, numerous clinical trials have investigated the role of multimodality therapy [15- 
17]. Surgical options for resection of esophageal carcinoma include transhiatal esophagectomy and transthoracic approaches, such as Ivor Lewis esophagectomy (abdominal and right thoracic approach, also called Lewis-Tanner esophagectomy), the three-incision modified McKeown esophagectomy that involves laparotomy, right thoracotomy and neck anastomosis, and left thoracotomy or a left thoracoabdominal approach [18-25]. The choice of surgical approach depends on location of the tumor and preference of the surgeon. All the procedures are complex and, therefore, treatment in high-volume centers with experienced surgeons and the availability of critical care support is associated with improved outcomes $[26,27]$. Furthermore, in junctional ACs - the 'true' cardia carcinomas - several surgical techniques are coexisting. Since the seventh edition of the American Joint Committee on Cancer/ International Union Against Cancer (AJCC/UICC) redefined the junctional ACs in 2009, a retrospective evaluation of surgical outcomes after both procedures becomes even more difficult $[28,29]$. Additional debate exists on the extension of lymphadenectomy (two vs. three fields), localization of the anastomosis (high intrathoracic vs. cervical) and hand versus stapler anastomoses.

There has been much recent debate regarding the best surgical procedure to treat EC, in particular with respect to the optimum extent of lymphadenectomy to improve survival while minimizing morbidity. Lymphadenectomy deserves to be given particular consideration in esophageal surgery. Cancer of the esophagus is a very lethal disease with a high case fatality ratio, and the opportunity to cure a patient is highly dependent on lymph node spread and its complete removal [30].

\section{Lymph Node Dissection}

Lymph node status is one of the most important prognostic factors for EC. It has already been established that lymph node metastasis from EC may occur at an early stage and skip to nodes far away from the primary tumor [30-32]. Upon presentation with dysphagia, over $70 \%$ of patients will already have lymph node metastasis. Of those who survive surgery, $80 \%$ will eventually die from tumor recurrence; at least $40 \%$ of these are due to recurrence in lymph nodes $[33,34]$. Theoretically, if all the involved regional nodes could be completely removed along with the tumor itself, the chance of cure would be increased and the risk of early locoregional recurrence reduced significantly.

Three-field lymphadenectomy (abdomen, chest and neck) is mainly practiced in Asia where esophageal SCC predominates [35]. In Europe and the USA where esophageal AC is common, this approach has few proponents and two-field lymphadenectomy in the abdomen and chest is more commonly used [22, 35]. Kato et al. [32] compared long-term survival between two groups with two- and three-field dissection. It was concluded that the survival rate was significantly better in patients with extensive three-field dissection than two-field dissection. Esophagectomy with three-field lymph node dissection can be performed with a low mortality and reasonable morbidity. An excellent overall survival suggests a superiority of three-field lymphadenectomy when performed at experienced centers [36]. Altorki et al. [22] reported that unsuspected metastases to the recurrent laryngeal and/or cervical nodes are present in $36 \%$ of patients regardless of cell type or location within the esophagus. $30 \%$ of patients were upstaged, mainly from stage III to stage IV. An overall 5-year survival rate of $51 \%$ suggests a true survival benefit beyond that achieved solely on the basis of stage migration [35].

However, a number of studies indicated that extended three-field lymphadenectomy was associated with a marked increase in recurrent nerve injuries varying between $15 \%$ and even as much as $70 \%[37,38]$. As a result, surgeons in the Western world remained reluctant to apply such extensive lymph node dissection, especially in a population in whom AC rather than SCC has become the predominant tumor type. Moreover, in the Western world, patients 
with tumors located above the level of the tracheal bifurcation are usually submitted to multimodality therapy, i.e. induction chemo- and/or radiotherapy followed by surgery. Therefore, data on three-field lymphadenectomy coming from Western centers are scarce. Lerut et al. [39] analyzed a series of 174 patients who underwent three-field lymphadenectomy. $23 \%$ of the patients with AC (25.8\% distal third and $17.6 \%$ gastroesophageal junction) and $25 \%$ of the patients with SCC (26.2\% middle third) had positive cervical nodes resulting in a change of pTNM staging specifically related to the unforeseen cervical lymph node involvement in $12 \%$. Cervical lymph node involvement was unforeseen in $75.6 \%$ of patients with cervical nodes at pathologic examinations. Five-year survival for patients with positive cervical nodes was $27.7 \%$ for middle third SCC. For distal third ACs, 4-year survival was $35.7 \%$ and 5 -year survival $11.9 \%$. A 5-year survival of $27.2 \%$ in patients with positive cervical nodes in middle third carcinomas indicates that these nodes should be considered as regional (N1) rather than distant metastasis (M1b) in middle third carcinomas. These patients seem to benefit from three-field lymphadenectomy. The role of three-field lymphadenectomy in distal third AC remains investigational.

Although there is now less doubt about the benefits of extended lymph node dissection, the optimal extent of dissection has always been controversial [40-42]. The greater the extent of dissection, the better the prognosis and local control might be, but the higher would be the surgical risks. In fact, complications directly related to surgical maneuvers, such as recurrent nerve palsy and anastomotic leakage, are far more common after three-field dissection than after two-field dissection [41]. All these have become major obstacles to an even wider application of extended nodal dissection. Therefore, a reasonable selection of dissection extent so as to diminish the surgical risks while still assuring the completeness of resection is the key to the problem. Attempts have already been made in several ways to pursue selective approaches in three-field lymphadenectomy. Early superficial cancers confined to the mucosa seldom metastasize to cervical nodes, and thus could be exempted from cervical dissection [43-45]. Fang et al. [46] proposed selective neck dissection based on cervical ultrasonography because the sensitivity and accuracy of ultrasonography for impalpable cervical lymphadenopathy could be as high as $80-90 \%$. The yield of metastatic cervical nodes $(19.5 \%)$ was in the same range as in the series of Tachimori et al. [47] (22.9\%) where all patients received neck dissection irrespective of the result of ultrasonography.

\section{Minimally Invasive Esophagectomy}

Esophagectomy is a highly invasive surgery and thus is associated with significant morbidity and mortality [48, 49]. Because of the potential advantages, including avoiding thoracotomy and laparotomy and reducing the rate of pulmonary infections (thus reducing inpatient stay), Cuschieri et al. [50] in 1992 reported the first series of five patients who underwent thoracoscopic mobilization of the thoracic esophagus. Akaishi et al. [51] described the results of thoracoscopic en bloc esophagectomy with radical mediastinal lymphadenectomy in Japan in 1996. Watson et al. [52] reported totally endoscopic Ivor Lewis esophagectomy in 1999. Nguyen et al. [53] described the combined laparoscopic and thoracoscopic approach to esophagectomy in the same year. Kernstine et al. [54] reported robot-assisted thoracoscopic esophagectomy in 2004. Up to now, several different approaches and patient positions for thoracoscopic esophagectomy have been reported. At present, more and more centers accept the semi-prone or prone position. The mediastinal view can be maintained by gravity in the prone position. Low-pressure carbon dioxide insufflation depresses the right lung anteriorly, maximizing exposure of the intrathoracic esophagus [55, 56]. Minimally invasive esophagectomy (MIE) was introduced into clinical practice in Taiwan in 1992 at the same time as in Western countries. MIE was gradually implemented and is now a commonplace procedure in the People's Republic of China [57-60]. Surgical approaches for MIE 
performed by Chinese cardiothoracic surgeons are multiple and complicated. The majority of centers use mainly total MIE (laparoscopic and thoracoscopic esophagectomy), whereas hybrid MIE (thoracoscopy and laparotomy/laparoscopy and thoracotomy) is used in routine practice in some centers. At our center, we originally used minimally invasive modified McKeown three-incision total MIE (laparoscopic and thoracoscopic esophagectomy) with three-field lymphadenectomy in 2009.

Minimally invasive surgery using the laparoscopic or thoracoscopic approach has been developed for various abdominal and thoracic procedures to minimize both surgical injury and invasiveness [61-63]. Minimally invasive approaches have also been incorporated into EC surgery in an attempt to improve postoperative outcomes of esophagectomy. Several MIE techniques have been described and represent safe alternatives for the surgical management of EC in centers with high volume and surgeons experienced in MIE. MIE has also been associated with less blood loss, less postoperative pain and shorter length of stay in the intensive care unit and hospital [64]. Luketich et al. [65] reviewed more than 1,000 patients treated with minimally invasive surgery for EC. The authors were able to show that the minimally invasive approach is technically high-demanding but feasible with an acceptable oncologic quality. Furthermore, reduced recurrent laryngeal nerve injury and mortality rates were revealed. There are little survival data available for MIE in China. Feng et al. [66] reported median survivals of 34.4 and 36.8 months for patients with EC after thoracoscope-assisted transthoracic esophagectomy and mediastinoscope-assisted transhiatal esophagectomy, respectively.

Up to now, total MIE using a combined thoracoscopic and laparoscopic approach can be performed safely, although the benefits for short-term outcomes are still controversial. Apart from favorable oncologic outcomes, MIE may have an advantage over open esophagectomy in lymph node dissection. However, the benefits of MIE still need to be confirmed by randomized controlled trials.

\section{Non-Surgical Treatment}

\section{Neoadjuvant Therapy}

Despite the optimization of surgical treatment and the formation of high-volume centers, the outcome following resection for EC remains unsatisfactory [67]. Therefore, preoperative (neoadjuvant) therapy has been established in order to tackle systemic disease as early as possible in the course of treatment and to shrink tumors in order to improve their resectability. Meanwhile, neoadjuvant chemotherapy and neoadjuvant chemoradiation have demonstrated a proven benefit for EC and can be recommended in patients in locally advanced stages $[5,68]$. Six fully published studies and one Cochrane review on published and unpublished studies have been performed to prove the benefit of neoadjuvant radiation without chemotherapy in resectable EC. Two studies reported a worse outcome in patients who had received neoadjuvant radiation. A Cochrane review on 1,147 patients, presenting mostly data on esophageal SCC patients who were randomized to neoadjuvant radiation or esophagectomy alone, concludes that neoadjuvant radiation led to a relative risk reduction for the endpoint death of $11 \%$ with a hazard ratio of 0.89 and a $95 \%$ confidence interval of $0.78-1.01$. The absolute survival difference was $2 \%$ after 2 years and $4 \%$ after 5 years. This meta-analysis revealed a trend toward improved 5-year overall survival but failed to show a statistically significant survival advantage [69]. Neoadjuvant radiation alone followed by surgery is therefore not a recommended approach to treat EC.

Two large studies included patients with esophageal SCC and esophageal AC while some smaller studies focused on either of these two histologic subtypes [16, 70]. Newer studies 
focusing on patients with esophageal AC show a significant survival benefit for patients undergoing neoadjuvant chemotherapy with cisplatin and 5-fluorouracil for 6-8 weeks [71]. A recently published meta-analysis of 2,062 patients who were randomly assigned to receive neoadjuvant chemotherapy or surgery alone showed no statistical significance for patients with esophageal SCC [53]. The effectiveness is unsatisfactory in patients with esophageal SCC, while in patients with esophageal AC neoadjuvant chemotherapy leads to a significant and clinically meaningful survival improvement without compromising the safety of surgical resection.

In recent years, neoadjuvant chemoradiation has been the most commonly investigated approach in the treatment of resectable EC. The recently published Chemoradiotherapy for Oesophageal Cancer Followed by Surgery Study (CROSS) is a prospective randomized controlled trial comparing surgery alone with neoadjuvant chemoradiation followed by surgery. CROSS shows a significant survival benefit for neoadjuvant treatment. Postoperative mortality was not increased in the tri-modality arm. CROSS certainly defines a new standard of care for the management of localized esophageal SCC, which consisted of weekly carboplatin plus paclitaxel combined with radiation 41.1 Gy followed by surgery. For AC, the results from CROSS are less clear than for esophageal SCC. The benefit associated with neoadjuvant chemoradiation in esophageal AC was in the same range as shown for neoadjuvant chemotherapy without radiation in some other studies [72]. Therefore, the debate whether chemotherapy alone or chemoradiation should be used as neoadjuvant treatment in localized esophageal AC is not yet over. In summery, neoadjuvant chemoradiation has proven efficacy in localized esophageal carcinoma that holds true for both common histologic subtypes.

\section{Adjuvant Therapy}

To decrease local tumor recurrence, several randomized trials compared surgery alone with surgery followed by radiation therapy. Studies enrolled a heterogeneous population, including patients with positive celiac nodes (M1a per the AJCC Cancer Staging, 6th ed.) and R1 resection [73-75]. Investigators used higher doses of radiation with different fractionation. Radiation was delivered from 6 to 12 weeks following surgery. These trials had conflicting results, with three studies failing to demonstrate any survival benefits for adjuvant radiation and one trial showing a worse survival in patients who received radiation therapy. Another trial showed improvement in quality of life in the surgery-only arm compared with the adjuvant radiation arm [76]. A small number of randomized trials evaluated postoperative chemotherapy and surgery compared with surgery alone. Pouliquen et al. [77] found no improvement in survival with postoperative chemotherapy (cisplatin/5-fluorouracil) in patients with esophageal SCC.

\section{Definitive Chemotherapy or Concurrent Chemoradiation}

Many patients with esophageal carcinoma have metastases at diagnosis, and in these patients the goal is to prolong survival and to improve quality of life. Chemotherapy or chemoradiation is effective in around $50 \%$ of these patients [78].

\section{Future Directions}

The treatment of esophageal carcinoma remains challenging but is best approached by a multidisciplinary team. Refinement of staging techniques, including molecular staging, is needed to understand prognosis and to tailor therapy to individuals in order to achieve the best possible outcomes. Technological advances in minimally invasive surgery, endoscopic treatments and targeted agents are being investigated and will hopefully also improve 
outcomes. The present recommended therapeutic management is individualized multidisciplinary team approaches according to patients' staging and physiologic reserve. The goal of multidisciplinary team therapy is to maximize survival and quality of life and also to decrease postoperative complications. MIE should be optimal in surgical therapy in future decades.

\section{References}

1 Pennathur A, Gibson MK, Jobe BA, Luketich JD: Oesophageal carcinoma. Lancet 2013;381:400-412.

2 Mao WM, Zheng WH, Ling ZQ: Epidemiologic risk factors for esophageal cancer development. Asian Pac J Cancer Prev 2011;12:2461-2466.

- Enzinger PC, Mayer RJ: Esophageal cancer. N Engl J Med 2003;349:2241-2252.

4 Siegel R, Naishadham D, Jemal A: Cancer statistics, 2013. CA Cancer J Clin 2013;63:11-30.

5 Lordick F, Holscher AH, Haustermans K, Wittekind C: Multimodal treatment of esophageal cancer. Langenbecks Arch Surg 2013;398:177-187.

6 Uzunoglu FG, Reeh M, Kutup A, Izbicki JR: Surgery of esophageal cancer. Langenbecks Arch Surg 2013;398: 189-193.

7 Fujishiro M: Perspective on the practical indications of endoscopic submucosal dissection of gastrointestinal neoplasms. World J Gastroenterol 2008;14:4289-4295.

8 Oyama T, Miyata Y, Shimaya S: Lymph nodal metastasis of m3, sm1 esophageal cancer (in Japanese). Stomach Intestine 2002;37:71-74.

-9 Hajime I, Naoyuki Y, Hitomi M, Kazuhiko N: Management of complications associated with endoscopic submucosal dissection/endoscopic mucosal resection for esophageal cancer. Dig Endosc 2013;25(suppl 1):29-38.

10 Ell C, May A, Pech 0, et al: Curative endoscopic resection of early esophageal adenocarcinomas (Barrett's cancer). Gastrointest Endosc 2007;65:3-10.

11 Altorki NK, Lee PC, Liss Y, et al: Multifocal neoplasia and nodal metastases in T1 esophageal carcinoma: implications for endoscopic treatment. Ann Surg 2008;247:434-439.

12 Fujishiro M, Yahagi N, Kakushima N, et al: Endoscopic submucosal dissection of esophageal squamous cell neoplasms. Clin Gastroenterol Hepatol 2006;4:688-694.

-13 Yamaguchi N, Isomoto H, Nakayama T, et al: Usefulness of oral prednisolone in the treatment of esophageal stricture after endoscopic submucosal dissection for superficial esophageal squamous cell carcinoma. Gastrointest Endosc 2011;73:1115-1121.

14 Hashimoto S, Kobayashi M, Takeuchi M, Sato Y, Narisawa R, Aoyagi Y: The efficacy of endoscopic triamcinolone injection for the prevention of esophageal stricture after endoscopic submucosal dissection. Gastrointest Endosc 2011;74:1389-1393.

15 Maki Y, Jill MW, Richard CK, Kenneth LM: Minimally invasive surgery for esophageal cancer: review of the literature and institutional experience. Cancer Control 2013;2:130-137.

16 Kelsen DP, Ginsberg R, Pajak TF, et al: Chemotherapy followed by surgery compared with surgery alone for localized esophageal cancer. N Engl J Med 1998;339:1979-1984.

17 Bosset JF, Gignoux M, Triboulet JP, et al: Chemoradiotherapy followed by surgery compared with surgery alone in squamous-cell cancer of the esophagus. N Engl J Med 1997;337:161-167.

18 Pennathur A, Luketich JD: Resection for esophageal cancer: strategies for optimal management. Ann Thorac Surg 2008;85:S751-S756.

19 Pennathur A, Zhang J, Chen H, Luketich JD: The ‘best operation' for esophageal cancer? Ann Thorac Surg 2010; 89:S2163-S2167.

20 Hagen JA, DeMeester SR, Peters JH, Chandrasoma P, DeMeester TR: Curative resection for esophageal adenocarcinoma: analysis of 100 en bloc esophagectomies. Ann Surg 2001;234:520-530; discussion 530-531.

21 Orringer MB, Marshall B, Iannettoni MD: Transhiatal esophagectomy: clinical experience and refinements. Ann Surg 1999;230:392-400; discussion 400-403.

22 Altorki N, Kent M, Ferrara C, Port J: Three-field lymph node dissection for squamous cell and adenocarcinoma of the esophagus. Ann Surg 2002;236:177-183.

-23 Swanson SJ, Batirel HF, Bueno R, et al: Transthoracic esophagectomy with radical mediastinal and abdominal lymph node dissection and cervical esophagogastrostomy for esophageal carcinoma. Ann Thorac Surg 2001; 72:1918-1924; discussion 1924-1925.

24 Visbal AL, Allen MS, Miller DL, Deschamps C, Trastek VF, Pairolero PC: Ivor Lewis esophagogastrectomy for esophageal cancer. Ann Thorac Surg 2001;71:1803-1808.

25 Mathisen DJ, Grillo HC, Wilkins EW Jr, Moncure AC, Hilgenberg AD: Transthoracic esophagectomy: a safe approach to carcinoma of the esophagus. Ann Thorac Surg 1988;45:137-143.

26 Birkmeyer JD, Siewers AE, Finlayson EV, et al: Hospital volume and surgical mortality in the United States. N Engl J Med 2002;346:1128-1137.

27 Dimick JB, Pronovost PJ, Heitmiller RF, Lipsett PA: Intensive care unit physician staffing is associated with decreased length of stay, hospital cost, and complications after esophageal resection. Crit Care Med 2001;29: 753-758. 
Reeh M, Nentwich MF, von Loga K, et al: An attempt at validation of the seventh edition of the classification by the International Union Against Cancer for esophageal carcinoma. Ann Thorac Surg 2012;93:890-896.

29 Sobin LH, Gospodarowicz MK, Wittekind C; International Union against Cancer: TNM Classification of Malignant Tumours, ed 7. Chichester, Wiley, 2012.

30 Akiyama H, Tsurumaru M, Udagawa H, Kajiyama Y: Radical lymph node dissection for cancer of the thoracic esophagus. Ann Surg 1994;220:364-372; discussion 372-373.

31 Isono K, Sato H, Nakayama K: Results of a nationwide study on three-field lymph node dissection of esophageal cancer. Oncology 1991;48:411-420.

-32 Kato H, Tachimori Y, Watanabe H, et al: Lymph node metastasis in thoracic esophageal carcinoma. J Surg Oncol 1991;48:106-111.

33 Mandard AM, Chasle J, Marnay J, et al: Autopsy findings in 111 cases of esophageal cancer. Cancer 1981;48: 329-333.

34 Isono K, Onoda S, Ishikawa T, Sato H, Nakayama K: Studies on the causes of death for esophageal carcinoma. Cancer 1982;49:2173-2179.

35 Nishihira T, Hirayama K, Mori S: A prospective randomized trial of extended cervical and superior mediastinal lymphadenectomy for carcinoma of the thoracic esophagus. Am J Surg 1998;175:47-51.

-36 Mitsuo T, Shoichi K, Hiroshi Y, Muneaki S, Yasuhito T, Dipok K, Naofumi N: Clinical outcomes of extended esophagectomy with three-field lymph node dissection for esophageal squamous cell carcinoma. Am J Surg 2005;189:98-109.

37 Isono K, Ochiai T, Koide Y: Indications for extended three-field lymphadenectomy for esophageal cancer. Dis Esophagus 1994;7:147-150.

-38 Fujita H, Kakegawa T, Yamana H, et al: Mortality and morbidity rates, postoperative course, quality of life, and prognosis after extended radical lymphadenectomy for esophageal cancer: comparison of three-field lymphadenectomy with two-field lymphadenectomy. Ann Surg 1995;222:654-662.

-39 Lerut T, Nafteux P, Moons J, Coosemans W, Decker G, De Leyn P, van Raemdonck D, Ectors N: Three-field lymphadenectomy for carcinoma of the esophagus and gastroesophageal junction in 174 R0 resections: impact on staging, disease-free survival, and outcome: a plea for adaptation of TNM classification in upper-half esophageal carcinoma. Ann Surg 2004;240:962-972; discussion 972-974.

40 Kato H, Watanabe H, Tachimori Y, Iizuka T: Evaluation of neck lymph node dissection for thoracic esophageal carcinoma. Ann Thorac Surg 1991;51:931-935.

41 Udagawa H, Akiyama H: Surgical treatment of esophageal cancer: Tokyo experience of the three-field technique. Dis Esophagus 2001;14:110-114.

42 Law S, Wong J: Two-field dissection is enough for esophageal cancer. Dis Esophagus 2001;14:98-103.

-43 Kato H, Tachimori Y, Mizobuchi S, Igaki H, Ochiai A: Cervical, mediastinal, and abdominal lymph node dissection (three-field dissection) for superficial carcinoma of the thoracic esophagus. Cancer 1993;72:2879-2882.

-44 Nakagawa S, Nishimaki T, Kosugi S, Ohashi M, Kanda T, Hatakeyama K: Cervical lymphadenectomy is beneficial for patients with carcinoma of the upper and mid-thoracic esophagus. Dis Esophagus 2003;16:4-8.

45 Noguchi T, Wada S, Takeno S, Hashimoto T, Moriyama H, Uchida Y: Two-step three-field lymph node dissection is beneficial for thoracic esophageal carcinoma. Dis Esophagus 2004;17:27-31.

46 Fang W, Chen W, Chen Y, Jiang Y: Selective three-field lymphadenectomy for thoracic esophageal squamous carcinoma. Dis Esophagus 2007;20:206-211.

47 Tachimori Y, Kato H, Watanabe H, Yamaguchi H: Neck ultrasonography for thoracic esophageal carcinoma. Ann Thorac Surg 1994;57:1180-1183.

48 Morita M, Yoshida R, Ikeda K, et al: Advances in esophageal cancer surgery in Japan: an analysis of 1,000 consecutive patients treated at a single institute. Surgery 2008;143:499-508.

-49 Jamieson GG, Mathew G, Ludemann R, Wayman J, Myers JC, Devitt PG: Postoperative mortality following oesophagectomy and problem in reporting its rate. Br J Surg 2004;91:943-947.

50 Cuschieri A, Shimi S, Banting S: Endoscopic oesophagectomy through a right thoracoscopic approach. J R Coll Surg Edinb 1992;37:7-11.

-51 Akaishi T, Kaneda I, Higuchi N, Kuriya Y, Kuramoto J, Toyoda T, Wakabayashi A: Thoracoscopic en bloc esophagectomy with radical mediastinal lymphadenectomy. J Thorac Cardiovasc Surg 1996;112:1533-1540.

52 Watson DI, Davies N, Jamieson GG: Totally endoscopic Ivor Lewis esophagectomy. Surg Endosc 1999;13:293297.

53 Nguyen NT, Schauer PR, Luketich JD: Combined laparoscopic and thoracoscopic approach to esophagectomy. J Am Coll Surg 1999;188:328-332.

54 Kernstine KH, DeArmond DT, Karimi M, et al: The robotic, 2-stage, 3-field esophagolymphadenectomy. J Thorac Cardiovasc Surg 2004;127:1847-1849.

-55 Fabian T, Martin J, Katigbak M, McKelvey AA, Federico JA: Thoracoscopic esophageal mobilization during minimally invasive esophagectomy: a head-to-head comparison of prone versus decubitus positions. Surg Endosc 2008;22:2485-2491.

56 Noshiro H, Iwasaki H, Kobayashi K, et al: Lymphadenectomy along the left recurrent laryngeal nerve by a minimally invasive esophagectomy in the prone position for thoracic esophageal cancer. Surg Endosc 2010;24: 2965-2973.

57 Liu HP, Chang CH, Lin PJ, Chang JP: Video-assisted endoscopic esophagectomy with stapled intrathoracic esophagogastric anastomosis. World J Surg 1995;19:745-747. 
58 Sabanathan S, Mearns AJ, Richardson J: Endoscopic oesophagectomy through a right thoracoscopic approach. J R Coll Surg Edinb 1992;37:284-285.

59 DePaula AL, Hashiba K, Ferreira EA, de Paula RA, Grecco E: Laparoscopic transhiatal esophagectomy with esophagogastroplasty. Surg Laparosc Endosc 1995;5:1-5.

60 Zingg U, McQuinn A, DiValentino D, et al: Minimally invasive versus open esophagectomy for patients with esophageal cancer. Ann Thorac Surg 2009;87:911-919.

61 Veldkamp R, Kuhry E, Hop WC, Jeekel J, Kazemier G, Bonjer HJ, Haglind E, Påhlman L, Cuesta MA, Msika S, Morino M, Lacy AM; COlon cancer Laparoscopic or Open Resection Study Group (COLOR): Laparoscopic surgery versus open surgery for colon cancer: short-term outcomes of a randomized trial. Lancet Oncol 2005; 6:477-484.

62 Kim HH, Hyung WJ, Cho GS, et al: Morbidity and mortality of laparoscopic gastrectomy versus open gastrectomy for gastric cancer: an interim report - a phase III multicenter, prospective, randomized trial (KLASS Trial). Ann Surg 2010;251:417-420.

63 Allen MS, Darling GE, Pechet TTV, et al: Morbidity and mortality of major pulmonary resections in patients with early-stage lung cancer: initial results of the randomized, prospective ACOSOG Z0030 trial. Ann Thorac Surg 2006;81:1013-1019.

64 Masayuki W, Yoshifumi B, Yohei N, Hideo B: Minimally invasive esophagectomy for esophageal cancer: an updated review. Surg Today 2013;43:237-244.

65 Luketich JD, Pennathur A, Awais 0, et al: Outcomes after minimally invasive esophagectomy: review of over 1,000 patients. Ann Surg 2012;256:95-103.

66 Feng MX, Wang H, Zhang Y, Tan LJ, Xu ZL, Qun W: Minimally invasive esophagectomy for esophageal squamous cell carcinoma: a case-control study of thoracoscope versus mediastinoscope assistance. Surg Endosc 2012; 26:1573-1578.

67 Mariette C, Piessen G, Triboulet JP: Therapeutic strategies in oesophageal carcinoma: role of surgery and other modalities. Lancet Oncol 2007;8:545-553.

68 Sjoquist KM, Burmeister BH, Smithers BM, et al: Survival after neoadjuvant chemotherapy or chemoradiotherapy for resectable esophageal carcinoma: an updated meta-analysis. Lancet Oncol 2011;12:681-692.

69 Arnott SJ, Duncan W, Gignoux M, Hansen HS, Launois B, Nygaard K, Parmar MK, Rousell A, Spilopoulos G, Stewart G, Tierney JF, Wang M, Rhugang Z; Oesophageal Cancer Collaborative Group: Preoperative radiotherapy for esophageal carcinoma. Cochrane Database Syst Rev 2005;4:CD001799.

-70 Medical Research Council Oesophageal Cancer Working Group: Surgical resection with or without preoperative chemotherapy in oesophageal cancer: a randomised controlled trial. Lancet 2002;359:1727-1733.

71 Ychou M, Boige V, Pignon JP, et al: Perioperative chemotherapy compared with surgery alone for resectable gastroesophageal adenocarcinoma: an FNCLCC and FFCD multicenter phase III trial. J Clin Oncol 2011;29: 1715-1721.

72 van Hagen P, Hulshof MC, van Lanschot JJ, et al: Preoperative chemoradiotherapy for esophageal or junctional cancer. N Engl J Med 2012;366:2074-2084.

73 Ténière P, Hay JM, Fingerhut A, Fagniez PL: Postoperative radiation therapy does not increase survival after curative resection for squamous cell carcinoma of the middle and lower esophagus as shown by a multicenter controlled trial. French University Association for Surgical Research. Surg Gynecol Obstet 1991;173:123-130.

74 Zieren HU, Müller JM, Jacobi CA, et al: Adjuvant postoperative radiation therapy after curative resection of squamous cell carcinoma of the thoracic esophagus: a prospective randomized study. World J Surg 1995;199: 444-449.

75 Fok M, Sham JS, Choy D, et al: Postoperative radiotherapy for carcinoma of the esophagus: a prospective, randomized controlled study. Surgery 1993;113:138-147.

76 Xiao ZF, Yang ZY, Liang J, et al: Value of radiotherapy after radical surgery for esophageal carcinoma: a report of 495 patients. Ann Thorac Surg 2003;75:331-336.

77 Pouliquen X, Levard H, Hay JM, et al: 5-Fluorouracil and cisplatin therapy after palliative surgical resection of squamous cell carcinoma of the esophagus: a multicenter randomized trial. French Associations for Surgical Research. Ann Surg 1996;223:127-133.

78 Polednak AP: Trends in survival for both histologic types of esophageal cancer in US surveillance, epidemiology and end results areas. Int J Cancer 2003;105:98-100. 